\title{
超快超强激光及其科学应用发展趋势研究
}

\author{
刘军，曾志男，梁晓燕，冷雨欣，李儒新 \\ (中国科学院上海光学精密机械研究所, 上海 201800)
}

\begin{abstract}
摘要: 超快超强激光兼具超快时域特性和超高峰值功率特性, 为人类在实验室中创造出了前所未有的超快时间、超高强场、 超高温度和超高压力等极端物理条件, 成为用于拓展人类认知的前沿基础科学研究最重要的工具之一。本文从超快激光和超 强激光的应用与发展需求出发, 系统调研了国内外研究和科学应用的现状, 提出了我国超快激光和超强激光的发展思路与目 标以及为实现这些目标需要重点发展的相关方法和技术。针对超高峰值功率和高重复频率超强激光面向未来的重要方向, 分析了我国分阶段发展的重点内容, 突出了相关技术与配套元器件研究的重要性。此外, 在注重基础研究、多方面吸引和培 育人才、加强国际合作、促进产业化等方面提出了超快超强激光发展的措施建议，以期为我国激光技术与科学应用的稳步发 展提供方向参照。
\end{abstract}

关键词: 超快超强激光; 飞秒激光; 阿秒激光; 拍瓦激光; 发展趋势

中图分类号: 0437 文献标识码: A

\section{Development Trend of Ultrafast and Ultraintense Lasers and Their Scientific Application}

\author{
Liu Jun, Zeng Zhinan, Liang Xiaoyan, Leng Yuxin, Li Ruxin
}

(Shanghai Institute of Optics and Fine Mechanics, Chinese Academy of Sciences, Shanghai 201800, China)

\begin{abstract}
Ultrafast and ultraintense lasers have ultrafast temporal and ultraintense focal intensity properties. They can create unprecedentedly extreme experimental conditions with ultrahigh time resolution, temperature, and pressure as well as ultrahighstrength field. The ultrafast and ultraintense laser is one of the important tools for frontier fundamental research meant for extending the knowledge of mankind. This paper starts with the analysis of the application and development demands of the ultrafast and ultraintense lasers and then systematically investigates the research status of these lasers in China and abroad. Based on this, the developing routes and targets of ultrafast and ultraintense lasers as well as the related technologies needed for achieving these targets are proposed. Particularly, we proposed the respective key steps for developing ultraintense lasers with ultrahigh peak power and those with high repetition frequency, and emphasized the study of related technologies and ancillary components. Furthermore, several suggestions are proposed for the development of ultrafast and ultraintense lasers in China, including strengthening fundamental researches, improving personnel training, enhancing international cooperation, and promoting market application, hoping to provide references for the steady development of China's laser technologies.
\end{abstract}

Keywords: ultraintense and ultrashort laser; femtosecond laser; attosecond laser; petawatt laser; development trend

收稿日期 : 2020-03-19; 修回日期 : 2020-04-22

通讯作者: 李儒新, 中国科学院上海光学精密机械研究所研究员, 中国科学院院士, 研究方向为超高峰值功率激光、超短脉冲激光与强 场激光物理; E-mail: ruxinli@mail.siom.ac.cn

资助项目：中国工程院咨询项目“我国激光技术与应用 2035 发展战略研究” (2018-XZ-27)

本刊网址：www.engineering.org.cn/ch/journal/sscae 


\section{一、前言}

激光出现后，依托锁模技术进入了飞秒 $\left(10^{-15} \mathrm{~s}\right)$ 超快时代, 并迅速应用到物理、生物、化学和材 料等前沿基础科学研究。Zewail 教授因飞秒化学 方面的开创性研究荣获 1999 年诺贝尔化学奖。啁 啾脉冲放大技术 (CPA) 进一步将激光推进到了超 强时代 [1], 相关科学家荣获 2018 年诺贝尔物理 学奖。

超快超强激光是指同时具有超快时域特性和超 高峰值功率特性的特殊光场, 为人类在实验室中创 造出了前所未有的超快时间、超高强场、超高温度 和超高压力等极端物理条件, 极大地促进了物理、 化学、生物、材料、医学以及交叉学科等前沿科学 的发展与进步。可以认为, 超快超强激光是用于拓 展人类认知的前沿基础科学研究最重要的工具之 一, 在某些方面甚至是独一无二、不可替代的研究 手段。

超快超强激光技术在推动前沿基础科学研究持 续拓展的同时, 又面临着前沿基础科学研究因自身 深化探索而新增的能力支撑需求，这为激光技术体 系发展赋予了强劲的诨引力。本文着重梳理超快超 强激光的发展与科学应用需求以及国内外技术发展 情况, 在此基础上就我国的领域发展目标和重点方 向开展论证分析，以期为我国激光技术的稳步发展 提供方向参照。

\section{二、超快超强激光应用与发展需求分析}

超快超强激光在相关前沿基础科学研究中的应 用拓展, 亟需进一步提升激光参数, 探索利用激 光脉冲的其他参量来将超快和超强前沿基础科学 研究推进到更为深入的物质层次。根据前沿科学 研究目标的差异, 未来领域应用与发展的需求集 中在以下两部分。

\section{（一）超快激光及其科学应用}

这一方向的未来发展需求可细分为阿秒激光乃 至入秒激光、极紫外 - 太赫兹全波段多维度参量精 密可控的飞秒超快激光。

阿秒激光乃至入秒激光追求采用更短脉冲宽度 的超快激光来研究物质内部更快的超快过程, 需要
发展更高脉冲能量、更短脉冲宽度、更高光子能量 的高性能阿秒 $\left(10^{-18} \mathrm{~s}\right)$ 激光。将阿秒脉冲的光子 能量推进到硬 X 射线波段和伽马射线波段，将脉冲 宽度推进到人秒 $\left(10^{-21} \mathrm{~s}\right)$ 的时间尺度，从而将人 类能够探索的物质层次从原子 / 分子水平推进到原 子核尺度 [2]。

飞秒时间尺度对应着原子 / 分子、材料、生物 蛋白、化学反应等丰富物质体系的超快过程, 有着 广泛而重要的应用。随着研究的进一步拓展与深入, 需要探索更加丰富和复杂的超快动力学过程, 以致 控制这些超快过程。为了对超快激光更多维度的参 量特性进行调制和利用，不仅需要将飞秒激光的光 谱拓展到红外 - 太赫兹波段、真空紫外 - 极紫外波 段, 还需要发展包括时域、振幅、相位、光谱、偏振、 空间模式等多维度参量在内的精密调控飞秒超快激 光, 以极紫外 - 太赫兹全波段多维度参量精密可控 的飞秒超快激光为代表。

\section{（二）超强激光及其科学应用}

根据定位和应用目标的差异，这一方向可分为 低重复频率超高峰值功率超强激光、高重复频率高 平均功率超强激光。其中，低重复频率是指激光脉 冲重复频率在 $10 \mathrm{~Hz}$ 及以下, 高重复频率是指激光 脉冲重复频率在 $1 \mathrm{kHz}$ 及以上。

唯有利用超强激光, 人类方可在实验室中产生 宇宙星体内部和原子核内部才有的极端物理条件。 利用低重复频率超高峰值功率超强激光, 可在实验 室中研究激光粒子加速、光核物理、伽马光 - 光对 撞等微观尺度的前沿物理问题，也可在宏观尺度上 研究超新星爆发、太阳耀斑、黑洞吸积盘喷流等天 体物理现象, 还可研究引力波、暗物质、真空物理 等拓展人类未知的前沿基础科学。针对国家重大理 论与实验研究的需求, 如激光粒子加速器、核嬗变 等核物理、高能物理、激光聚变能源新途径、激光 核医学等, 低重复频率超高峰值功率超强激光提供 了重要的科学研究工具。

在与国家战略需求相关的应用领域，如空天安 全、空天环境物理等方面, 高平均功率的超强激光 是重要的驱动工具, 以能够适应空天特殊环境的高 重复频率超强激光为典型。高重复频率高平均功率 的超强激光产生超强质子束、电子束、中子束、 $\mathrm{X}$ 射线、伽马射线, 以致超强太赫兹脉冲等次级超强 
光源作为新型工具, 可以拓展到光核反应、激光推 进、核聚变能源和核废料处理、疾病治疗等更为前 沿的重大基础科学研究和实际应用中。

\section{三、超快超强激光国内外研究现状}

\section{（一）超快激光及其科学应用}

\section{1. 阿秒超快激光}

近 20 年的发展历程表明, 宽带高次谐波产生 阿秒脉冲来拓展应用的根本局限在于单脉冲能量偏 低, 国际主流的解决途径是建立高功率和长波长的 飞秒超快激光系统。欧盟投资数亿欧元，在匈牙利 建立了极端光装置 - 阿秒脉冲源（ELI-ALPS），通 过两个拍瓦激光系统产生高峰值功率和高平均功率 的阿秒脉冲 [3]。长波长的中红外飞秒激光脉冲系 统可产生更高光子能量和更短脉冲宽度的阿秒脉 冲 [4], 因此众多研究机构均在这方面开展工作。高 重复频率阿秒激光研究也取得重要进展 [5]。另外, 通过 X 射线自由电子激光 (XFEL) 产生阿秒脉冲 也获得了初步验证, XFEL 在产生高光子能量（硬 $\mathrm{X}$ 射线和伽马射线波段）的高功率阿秒脉冲方面具 有一定优势。

国内阿秒激光研究集中在中国科学院所属的上 海光学精密机械研究所、物理研究所、西安光学精 密机械研究所等科研机构。由于总体布局较晚, 当 前研究水平仍然相对落后。2009 年, 上海光学精密 机械研究所测量了阿秒脉冲链的脉冲宽度, 获得了 近傅里叶变换极限的阿秒脉冲激光。2013 年, 物理 研究所产生并测量了单个阿秒脉冲, 获得了脉冲宽 度为 160 as 的脉冲激光。西安光学精密机械研究所 在阿秒脉冲激光研究方面承担了较多任务。国内高 等院校, 如华中科技大学、华东师范大学、北京大 学、国防科技大学等也在开展阿秒激光的相关研究。 此外, 一些研究机构还在高功率激光加速产生高能 电子和伽马射线等方面开展了系列工作。

\section{2. 飞秒超快激光}

利用非线性光学方法, 国际上早已将飞秒激光 的波长从可见 - 近红外波段拓展到深紫外-紫外、 红外 - 太赫兹波段。自由电子激光器也已获得真空 紫外和极紫外波段以及太赫兹超快飞秒激光, 具有 高能量和波长可调谐的优势, 但相关装置较为复杂。 为了研究更复杂丰富的超快动力学过程, 多参量光
场精密调控和多波长飞秒超快激光也获得了发展。

国内较多研究团队直接采用商用进口的飞秒激 光器, 叠加非线性效应来拓展波长等参量。在光场 精密调控和多波长飞秒超快激光方面, 上海光学精 密机械研究所、上海科技大学、西安交通大学等机 构完成了系列研究。2019 年, 中国科学院大连化学 物理研究所构建的自由电子激光器已经投入运行, 在 50 200 nm 真空紫外与极紫外波段实现了波长连 续可调的超快激光输出, 发挥了飞秒超快激光对基 础科学研究的支撑和拓展作用 [6]。中国工程物理 研究院利用自由电子激光实现了太赫兹波段超快激 光输出。

\section{（二）超强激光及其科学应用}

这一方向的国际研究进展快速且竞争激烈, 世 界上已建成 50 多套拍瓦级激光装置 [7]。

1. 低重复频率超高峰值功率超强激光

欧盟、美国、日本、韩国、俄罗斯等国家或地 区均在建设十拍瓦级激光重大科学装置。近期多个 国家或地区提出了 100 200 PW 重大激光科学装置 的发展计划。欧盟 10 多个国家的近 40 个科研机构 联合提出超强光基础设施（ELI）计划, 旨在发展 $200 \mathrm{PW}$ 超强激光装置, 已被纳入欧盟未来大科学 装置发展路线图; 2019 年实现了 $10 \mathrm{PW}$ 超强激光 输出 [8]。法国 Apollon 激光装置 [9] 2017 年实现了 $5 \mathrm{PW}$ 激光输出, 2018 年实现了 $10 \mathrm{PW}$ 激光输出, 更高指标输出目前有所延迟。英国 Vulcan 激光装 置 [10] 计划采用光参量啁啾脉冲放大 (OPCPA) 技 术, 将输出脉冲峰值功率由拍瓦级提升至十拍瓦级。 俄罗斯规划用于极端光学研究的艾瓦中心 (XCELS) 拟实现 $200 \mathrm{PW}$ 峰值功率, 待建激光装置包含 12 束 功率为 $15 \mathrm{PW}$ 、脉冲宽度为 $25 \mathrm{fs}$ 超强激光, 利用 相干合成技术来输出激光 [11]。日本激光快速点燃 实验项目（LFEX）装置已经实现了皮秒量级、脉 冲能量达 $2 \mathrm{~kJ}$ 的拍瓦激光输出, 主要用于支持快点 火激光核聚变、天体物理方面的研究。韩国光州科 学技术院（GIST）基于钛宝石 CPA 方案, 在 $0.1 \mathrm{~Hz}$ 重复频率下实现了 $4.2 \mathrm{PW}$ 激光输出 [12]。美国罗 彻斯特大学 OMEGA EP 装置具有 $1 \mathrm{~kJ} / 1 \mathrm{ps} / 1 \mathrm{PW}$ 的 激光输出能力, 同步提出了百拍瓦级超强激光的发 展构想。

国内低重复频率超高峰值功率超强激光研究 
方向起步较早，已经形成了实力较强、梯队合理的 研究队伍。自 1996 年起, 每两年召开 1 次的 “全 国强场激光物理会议” 显著促进了相关领域的学术 交流和研究进展。近年来, 我国在此方向取得了一 些重要研究成果, 部分成果已经处于国际领先水 平。2017 年, 中国工程物理研究院基于大口径三 硼酸锂（LBO）晶体和 OPCPA 技术路线获得了近 $5 \mathrm{PW}$ 超强激光输出 [13]。上海光学精密机械研究所 利用钛宝石 CPA 方案, 2016 年在国际上率先实现 $5 \mathrm{PW}$ 激光输出, 2017 年在国际上率先实现 $10 \mathrm{PW}$ 放大输出 [14]; 利用 OPCPA 技术也实现了 1 PW 激 光输出 [15]; 2018 年在国际上率先立项并启动建设 百拍瓦级超强激光装置。此外, 一些高等院校近期 也提出了建设数十拍瓦级激光装置的规划设想。

\section{2. 高重复频率高平均功率超强激光}

这一方向的技术方法主要分为碟片超快激光和 光纤超快激光。碟片激光器在解决增益介质的热效 应管理问题之后，实现了平均功率为千瓦级的输出。 光纤飞秒激光具有散热好、集成方便灵活、光束 质量好、转换效率高等优势, 且可实现 $1 \mathrm{MHz}$ 以 上重复频率的激光放大，近年来获得迅速发展。 受限于非线性效应, 光纤中的 CPA 输出能量和功 率还不高。

2012 年, 国际知名学者 Mourou 教授在欧盟组 织启动了 “国际放大相干网络” (ICAN) 计划 [16], 旨在推动基于光纤飞秒激光及其组束技术的发展, 实现高重复频率、高平均功率和高峰值功率的超强 激光脉冲, 探索应用于新一代粒子加速器的驱动源。 在 ICAN 计划 ( $10 \mathrm{~J} / 100 \mathrm{fs} / 10 \mathrm{kHz}$ 超强激光)框架下, 德国耶拿大学牵头完成了光纤飞秒激光时间与空间 组束的众多研究。例如, 已经采用 16 束光纤飞秒 激光合束获得了平均功率为千瓦级的高重复频率激 光输出; 提出的空间相干组束 $(16 \times 32)$ 与时间相 干组束或脉冲堆积相结合的新技术方案, 有望更加 经济地实现 $300 \mathrm{fs} / 100 \mathrm{TW}$ 超强激光输出 [17]。

国内高重复频率高平均功率超强激光还缺乏系 统的研究布局, 仅有上海光学精密机械研究所、北 京大学、国防科技大学、天津大学等少量研究单位 各自在分立的核心技术方向上开展研究和探索, 如 高性能增益光纤研制、碟片激光放大技术、光纤飞 秒振荡器、光纤 CPA 技术、空间激光组束、脉冲 时间堆积和脉冲压缩等。一些科研机构和高等院
校对大模场面积增益光纤、高能量高功率飞秒激 光等技术方向进行了持续研究。鉴于在微加工领域 应用的良好前景，国内诸多企业开展了数十瓦功 率的光纤飞秒激光产品研制, 部分企业已经推出了 功率为 $50 \mathrm{~W}$ 及以上的飞秒超快激光产品。尽管发 展迅速, 但大多数产品需要采用国外的关键器件, 而具有自主知识产权的关键器件还较少。整体来看, 这方面的研究较为分散, 尚未在产业链条上形成系 统规划和分工协作的局面。

\section{四、我国超快超强激光发展思路与目标}

\section{（一）超快激光及其科学应用}

\section{1. 阿秒超快激光}

阿秒脉冲的光子能量突破至 $1 \mathrm{keV}$ 乃至 $10 \mathrm{keV}$ 水平，支持开展阿秒超快内壳层电子动力学、电子 自旋 - 轨道动力学等基础物理过程、大分子乃至生 物大分子等复杂结构的超快电子动力学与结构变化 等研究。涉及的关键技术包括: 高功率、少周期、 载波包络相位稳定的中红外激光系统, 高亮度千电 子伏特级阿秒激光脉冲产生, 高分辨电子与多电子 动量测量, 通过康普顿散射方法将光子能量推进到 硬 X 射线波段和伽马射线波段。

超快脉冲的脉冲宽度突破至人秒水平，支持开 展深内壳层电子动力学乃至原子核的动力学研究。 阿秒脉冲的光子能量达到 $10 \mathrm{keV}$ 水平乃至伽马射 线波段，阿秒脉冲宽度具备进入入秒时间尺度的可 能性。涉及的关键技术包括：与提高产生效率相关 的技术，与实际应用相关的超快测量技术， 入秒脉 冲宽度测量等。

\section{2. 飞秒超快激光}

随着飞秒超快光谱基础科学研究的发展, 除了 利用脉冲时域特性以外, 光谱和偏振特性也是可以 利用的重要特性。后续主要研究思路为: 发展兆赫 兹重复频率极紫外 - 太赫兹波段宽带飞秒激光, 发 展高性能、多波长的飞秒激光脉冲和多波长飞秒光 频梳，实现同时脉冲形状和空间径向偏振（或浴旋） 的、精密调控的特殊时空结构飞秒激光; 发展吉赫 兹重复频率超快激光, 突破单光子和量子纠缠等新 型超快光谱技术，提升超快光谱的稳定性和探测效 率，支持更加纯粹的微观体系和更加复杂的多体超 快动力学过程研究; 利用多参量精密可控的超快激 
光, 研究脑科学、肿瘤、生物发育与再生等方面的 生物过程精密光控制。

\section{（二）超强激光及其科学应用}

1. 低重复频率超高峰值功率超强激光

需求牵引在于重大前沿物理科学问题研究, 以 期拓展人类认知。后续发展方向依然是继续提升激 光的峰值功率 (从 $100 \mathrm{PW}$ 到 $1 \mathrm{EW}$ ), 抢占最高聚 焦功率密度 $\left(10^{25} \mathrm{~W} / \mathrm{cm}^{2}\right)$ 的技术高地, 为科学前 沿研究提供最先进的极端物理条件。为了提升这类 前沿实验的效率和可靠性, 还应适当提升超强激光 的重复频率, 开展涡旋光等特殊光场的超强激光输 出及其应用研究; 时空电场精密控制与波长调谐的 超强激光将进一步拓展应用范围。随着激光聚焦功 率密度的不断提升, 激光脉冲的时间对比度要求越 来越高, 应针对性开展有关输出与测量的创新研究。 此外, 大口径激光聚焦的创新研究和设计成为发展 函需, 在有效提升聚焦功率密度的同时, 可缓解放 大输出激光能量伴生的成本问题。

峰值功率和重复频率是未来研究发展的突破 口。预计在 2025 年、 2030 年和 2035 年, 将分别实 现 $100 \mathrm{PW} 、 500 \mathrm{PW}$ 和 $1000 \mathrm{PW}$ (1 EW) 峰值功 率的激光输出, 在重复频率方面也将取得突破性提 升。(1)利用 5 年左右的时间, 实现单发 $100 \mathrm{PW}$ 峰 值功率输出、重复频率 $10 \mathrm{PW}$ 激光输出; 激光装置 进行真空极化处理，支持天体物理、反物质等基础 研究初步取得开创性科研成果。(2)利用 10 年左右 的时间, 通过提升泵浦激光能量来突破大尺寸光棚 等关键元器件的研制和延寿问题, 利用空间激光合 束等方法实现 $500 \mathrm{PW}$ 激光输出, 支持开展引力波、 暗物质等前沿重大研究。(3)利用 15 年左右的时间, 在更高功率泵浦激光方面, 通过提升大尺寸光梛等 关键元器件的尺寸和损伤阈值, 结合空间相干组束 方法来实现艾瓦级激光输出; 发展新型聚焦系统, 将聚焦功率密度提升至 $10^{25} \mathrm{~W} / \mathrm{cm}^{2}$; 探索基于光和 物质相互作用的新原理、新方法来实现艾瓦级激光 输出, 为激光发展开拓新的技术方案; 获得达到近 量子电动力学 (QED) 区域的超强激光, 支持开展 更加前沿的强场激光物理研究。

2. 高重复频率高平均功率超强激光

根据我国的现有技术水平、技术发展预期和国 家重大需求, 高重复频率高平均功率超强激光发展
具有以下发展趋势。(1)利用 5 年左右的时间, 重点 掌握飞秒光纤 CPA、空间相干组束、脉冲时间堆积、 大能量脉冲压缩等核心技术，通过路径和设计创 新, 降低这类激光的复杂性、难度和成本。(2)利用 10 年左右的时间, 在实验室中产生太瓦级千赫兹重 复频率的超强激光输出; 重点开展强场激光物理中 的高次谐波产生阿秒激光脉冲、激光电子加速等研 究, 获得高通量的阿秒激光脉冲, 促进原子 / 分子 和材料中阿秒动力学研究的发展; 通过激光技术突 破来带动工业应用的大发展，降低光纤飞秒激光的 功率成本。(3)利用 15 年左右的时间, 实现十太瓦 级千赫兹以上重复频率的超强激光输出; 通过工业 领域的批量应用来驱动光纤飞秒激光功率成本的显 著降低; 对太瓦级激光进行空间合束，在实验室中 实现十太瓦级高重复频率的超强激光; 重点开展小 型化粒子加速器研究, 促进高重复频率、高能量质 子束在医疗领域的拓展应用; 利用激光产生的高能 中子源, 探索激光聚变能源和核废料处理等重要方 面的应用。

\section{五、超快超强激光的重点技术方向}

\section{1. 阿秒超快激光}

未来重点发展方向主要包括: 高能量单个阿秒 激光脉冲, 高平均功率 (高重复频率) 阿秒激光, 高光子能量阿秒脉冲, 拓展阿秒脉冲应用的小型化 高重复频率阿秒脉冲。相关的技术发展方向为：高 品质的少周期（含中红外）激光脉冲技术，简单便 捷的阿秒激光脉冲测量技术、新型阿秒激光应用技 术, 高品质高亮度硬 $\mathrm{X}$ 射线和伽马射线产生技术、 入秒激光技术等。

\section{2. 飞秒超快激光}

未来重点发展方向主要包括: 多波长高性能飞 秒激光技术，宽带双频 / 多频梳飞秒激光技术，兆 赫兹高重复频率高性能真空紫外 - 极紫外、红外 太赫兹超快激光技术, 径向偏振和浴旋等特殊偏振 与空间模式的飞秒激光技术，吉赫兹高重复频率小 型化量子点超快激光技术，垂直腔面发射（VCSEL） 超快激光技术，涉及时域、光谱、偏振、空间、相 位和振幅等多维度光场精密调控的飞秒激光技术等。

3. 低重复频率超高峰值功率超强激光

聚焦功率密度、对比度是最重要的参数指标, 
应进一步发展放大技术、脉冲压缩技术、空间聚焦 技术、对比度提升与测量技术。未来重点技术方向 具体包括: 高通量放大技术 (即超大能量的 CPA 或 OPCPA 技术以及对应的超大口径激光晶体或非 线性晶体研制)，等离子体拉曼放大和准参量啁啾 脉冲放大（QPCPA）等新型放大技术，新型压缩器 设计及大口径、高损伤阈值压缩光栅的研制，大口 径超强激光组束技术, 激光脉冲对比度提升与单发 测量技术，大口径超强激光时空特性在线测量技术， 大口径超强激光波前整形与新型高性能聚焦系统设 计, 超强激光时空电场精密控制与波长调谐技术, 超强激光脉冲的腔外脉冲压缩技术，浴旋、径向偏 振等特殊光场的超强激光产生及其应用等。

\section{4. 高重复频率高平均功率超强激光}

未来重点发展方向主要包括: 新型飞秒光纤放 大、新型碟片激光放大技术，高重复频率飞秒激光 脉冲时间堆积与空间相干组束技术及其衍生创新技 术，空间相干组束中甚多束激光的相位测量与主动 反馈控制技术，新型飞秒激光放大的特殊光纤设计 与加工技术，脉冲压缩与色散管理技术，高重复频 率激光洜浦源技术，高重复频率放大过程中热效应 管理技术，高性能增益光纤、高性能啁啾光纤光栅 与透射光栅等核心元器件研制, 时空光场精密控制 与波长调谐技术等。

\section{六、对策建议}

(1) 结合国家在太空与深空、医疗、能源、核 物理等领域的远期重大需求, 真空物理、暗物质、 引力波等国际重大基础科学前沿的探索需要, 尽快 制定中长期发展战略规划, 成立超快超强激光基础 科学中心或协同创新中心，为相关重要研究方向提 供持续稳定的资源保障。注重基础研究, 追求实 现更多 “从 0 到 1 ” 的原创性突破，加强核心技术 和 “卡脖子” 技术的梳理分类, 提前进行科学布局 和技术攻关。

（2）基础科学的突破发展，重在具有原创和创 新能力的科研人员。鼓励创造有利于创新的科研环 境氛围, 建立有利于创新的科研管理与激励制度。 引进和培育更多数量的领域科研人才，特别是具有 原创性科研思想和交叉学科背景的领军人才。以人
才发展保障众多原创性成果涌现，从而引领世界激 光领域的科学和技术研究。

(3) 提高人类认知的基础科学研究, 不仅需要 本国科研人员的创新创造, 还需要全球科学家的聪 明才智。加强国际交流合作，吸引国际性人才开展 联合研究, 进一步加速和提升相关科学研究。在超 强激光这些我国已经处于领先地位的领域方向以及 一些具有引领性、颠覆性创新的研究方向, 可以 考虑在 “一带一路” 倡议框架下，开展重大基础 科学装置建设, 以我国为主并吸引其他国家 (如 亚洲国家、俄罗斯等) 开展联合研究和技术攻关。 通过基础科研成果共享 (类似 ELI 计划和黑洞探测 计划等）来提升我国科技创新的国际影响力。

（4）为了更好更快实现基础研究成果服务于国 家经济社会发展需求的目标，建议科研机构和高等 院校加强与企业的合作，促进超快超强激光方面实 用型科技成果的高效转化。同时加强知识产权保护 与管理，做好技术风险防范工作。

\section{参考文献}

[1] Strickland D, Mourou G. Compression of amplified chirped optical pulses [J]. Optics Communications, 1985, 56(3): 219-221.

[2] Krausz F, Ivanov M. Attosecond physics [J]. Review of Modern Physics, 2009, 81(1): 163-234.

[3] Kühn S, Dumergue M, Kahaly S, et al. The ELI-ALPS facility: The next generation of attosecond sources [J]. Journal of Physics B: Atomic, Molecular and Optical Physics, 2017, 50(13): 1-39.

[4] Popmintchev T, Chen M, Popmintchev D, et al. Bright coherent ultrahigh harmonics in the $\mathrm{keV} \mathrm{X}$-ray regime from mid-infrared femtosecond lasers [J]. Science, 2012, 336: 1287-1291.

[5] Saule T, Heinrich S, Schötz J, et al. High-flux ultrafast extreme-ultraviolet photoemission spectroscopy at $18.4 \mathrm{MHz}$ pulse repetition rate [J]. Nature Communications, 2019, 10(1): 1-10.

[6] 余永, 李钦明, 杨家岳, 等. 大连极紫外相干光源 [J]. 中国激光, 2019, 46(1): 35-42.

Yu Y, Li Q M, Yang J Y, et al. Dalian extreme ultraviolet coherent light source [J]. Chinese Journal of Lasers, 2019, 46(1): 35-42.

[7] Danson C N, Haefner C, Bromage J, et al. Petawatt and exawatt class lasers worldwide [J]. High Power Laser Science and Engineering, 2019, 7(3): 1-54.

[8] Gales S, Tanaka K A, Balabanski D L, et al. The extreme light infrastructure-nuclear physics (ELI-NP) facility: New horizons in physics with $10 \mathrm{PW}$ ultra-intense lasers and $20 \mathrm{MeV}$ brilliant gamma beams [J]. Reports on Progress in Physics, 2018, 81(9): $1-31$.

[9] Papadopoulos D N, Zou J P, Le Blanc C, et al. The Apollon 10 PW laser: Experimental and theoretical investigation of the temporal characteristics [J]. High Power Laser Science and Engineering, 
2016, 4(E34): 127-133.

[10] Hernandez-Gomez C, Blake S P, Chekhlov O, et al. The vulcan $10 \mathrm{PW}$ project [C]. San Francisco: The Sixth International Conference on Inertial Fusion Sciences and Applications, 2009.

[11] Shaykin A, Kostyukov I, Sergeev A, et al. Prospects of PEARL 10 and XCELS laser facilities [J]. The Review of Laser Engineering, 2014, 42:141-144.

[12] Sung J H, Lee H W, Yoo J Y, et al. 4.2 PW, 20 fs Ti:sapphire laser at $0.1 \mathrm{~Hz}$ [J]. Optics Letters, 2017, 42(11): 2058-2061.

[13] Zeng X M, Zhou K N, Zuo Y L, et al. Multi-petawatt laser facility fully based on optical parametric chirped-pulse amplification [J]. Optics Letters, 2017, 42(10): 2014-2017.
[14] Li W Q, Gan Z B, Yu L H, et al. 339 J high-energy Ti:sapphire chirped-pulse amplifier for $10 \mathrm{PW}$ laser facility [J]. Optics Letters, 2018, 43(22): 5681-5684.

[15] Yu L H, Liang X Y, Xu L, et al. Optimization for high-energy and high-efficiency broadband optical parametric chirped-pulse amplification in LBO near $800 \mathrm{~nm}[\mathrm{~J}]$. Optics Letters, 2015, 40(14): 3412-3415.

[16] Mourou G, Brocklesby B, Tajima T, et al. The future is fibre accelerators [J]. Nature Photonics, 2013, 7: 258-261.

[17] Breitkopf S, Eidam T, Klenke A, et al. A concept for multiterawatt fibre lasers based on coherent pulse stacking in passive cavities [J]. Light: Science \& Applications, 2014, 3(E211): 1-7. 